\title{
SOME APPLICATIONS OF FRACTIONAL CALCULUS TO OPERATOR SEMIGROUPS AND FUNCTIONAL CALCULUS
}

\author{
JOSÉ E. GALÉ \\ Departamento de Matemáticas, Universidad de Zaragoza \\ E-50009 Zaragoza, Spain \\ E-mail: gale@unizar.es
}

\begin{abstract}
We survey some recent results on functional calculus for generators of holomorphic semigroups, which have been obtained using versions of fractional derivation of RiemannLiouville or Weyl type. Such a calculus allows us to give tight estimates even in concrete $L^{1}$ examples.
\end{abstract}

1. Introduction. Let $A$ be a closed, not necessarily bounded, operator on a Banach space $X$. A functional calculus for $A$ is intended as a method to define, bounded or unbounded, operators $f(A)$ on $X$, where $f$ is a function whose domain contains the spectrum $\sigma(A)$ of $A$, so that $A$ is to play formally the role of the natural variable of $f$. This idea is useful in several respects. Important notions naturally associated to $A$, such as semigroups, resolvent functions, fractional powers, logarithms, spectral families, may be presented in the form $f(A)$, which facilitates considerably their study, in some directions.

Functions operating in a functional calculus usually satisfy some reproducing formula suitably adapted to the problem. In a first stage or even in its final form, the calculus is defined using an operator-valued version of such a formula. A well-known example is the Dunford-Riesz calculus built on the classical Cauchy formula for holomorphic functions [HP], [CDMY].

In this paper, we are concerned with the representation of Riemann-Liouville type

$$
f(t)=\frac{(-1)^{[\nu]}}{\Gamma(\nu)} \int_{-\infty}^{\infty}(u-t)_{+}^{\nu-1} f^{(\nu)}(u) d u, \quad(t \in \mathbb{R}),
$$

2000 Mathematics Subject Classification: Primary 47A60, 47D03, 26A33; Secondary 47D06, 43A20, 43A22.

Key words and phrases: holomorphic semigroup, generator, functional calculus, Banach algebra, fractional calculus.

This work has been partially supported by Project BFM2001-1793, M.C.YT.D.G.I. and F.E.D.E.R., Spain, and Project E12/25, D.G. Aragón, Spain.

The paper is in final form and no version of it will be published elsewhere. 
where $\nu>0,[\nu]=$ integer part of $\nu,(u-t)_{+}^{\nu-1}=(u-t)^{\nu-1} \chi_{(t, \infty)}(u)$ if $t, u \in \mathbb{R}, f$ is a suitable function on $\mathbb{R}$, and $f^{(\nu)}$ is to mean a fractional derivative of $f$ (when $\nu$ is an integer, the formula is obtained just integrating by parts, for suitable $f$ ).

The reproducing formula (1.1) has been the basis for theorems on radial multipliers, or Jacobi and Hankel multipliers, see [CGT1], $[\mathrm{T}],[\mathrm{GT}]$ and references therein. For certain operators $A$ the expression $R_{\nu-1}(u, A):=\frac{1}{\Gamma(\nu)}(u-A)_{+}^{\nu-1}$ makes sense as a bounded operator on $X$, which we call the Bochner-Riesz kernel of $A$. So, replacing $t$ with $A$ in (1.1) provides us with bounded operators of the form $f(A)$.

An appropriate definition of $f^{(\nu)}$ is the following one. If $\nu=[\nu]+\delta, 0<\delta<1$, let us first define

$$
I_{\omega}^{\delta} f(t):=\left(\frac{1}{\Gamma(\delta)} \int_{t}^{\omega}(s-t)^{\delta-1} f(s) d s\right) \chi_{(0, \omega)}(t),
$$

if $\omega \geq 0, t \in \mathbb{R}$; and then the fractional derivatives

$$
f^{(\delta)}(t)=-\lim _{\omega \rightarrow \infty}(d / d t) I_{\omega}^{1-\delta} f(t)
$$

and

$$
f^{(\nu)}(t)=(d / d t)^{[\nu]} f^{(\delta)}(t),
$$

if $t>0$, whenever the right sides exist [T], [CGT].

Let $B V_{1}$ be the usual class of functions of bounded variation on $[0, \infty]$. For $\nu>0$ let $B V_{\nu+1}$ denote the class of functions in $C([0, \infty])$ for which $f^{(\delta)}, \ldots, f^{(\nu-1)}$ exist and are locally absolutely continuous on $(0, \infty)$, with $f^{(\nu)}$ locally of bounded variation on $(0, \infty)$ and such that $\int_{0}^{\infty} t^{\nu}\left|d f^{(\nu)}(t)\right|<\infty$. Then $f-f_{\infty}$ satisfies (1.1) for all $f \in B V_{\nu+1}$, where $f_{\infty}:=\lim _{t \rightarrow \infty} f(t)$. Under pointwise multiplication $B V_{\nu+1}$ is a Banach algebra with respect to the norm $\Gamma(\nu+1)^{-1} \int_{0}^{\infty} t^{\nu} d f^{(\nu)}(t)+f_{\infty}[\mathrm{T}]$. For $\mu \geq \nu \geq 1$ we always have $B V_{\mu} \subset B V_{\nu}$.

Suppose that $A$ is a closed, densely defined operator on $X$. In order to give a meaning to $R_{\nu}(u, A)$ it is usually assumed that there is a Hilbert space $H$ on which $A$ acts as a (formally) self-adjoint operator. Then the spectral theorem guarantees that $R_{\nu}(u, A)$ exists as a bounded operator on $H$. It is further assumed that $H \cap X$ is dense in $H$ and $X$ and that the restriction of $R_{\nu}(u, A)$ to $H \cap X$ can be extended as a bounded operator from $X$ into $X$. In this way we have the following theorem. Let $\mathcal{L}(X)$ denote the usual Banach algebra of bounded operators on $X$.

Theorem 1.1. Suppose that $\left\|R_{\nu}(u, A) x\right\|_{X} \leq C_{\nu} u^{\nu}\|x\|_{X}$ for all $u>0$ and $x \in H \cap X$. Then the correspondence $f \mapsto f(A), B V_{\nu+1} \rightarrow \mathcal{L}(X)$ defined by

$$
f(A) x:=(-1)^{[\nu]+1} \int_{0}^{\infty} R_{\nu}(u, A) x d f^{(\nu)}(u)+f_{\infty} I_{d}, \quad(x \in X)
$$

is a bounded algebra homomorphism.

This result is given in [BNT, Theorem 2] in terms of spectral multipliers to be found in approximation processes. The present statement follows readily from observations in $[\mathrm{BNT}]$ and $[\mathrm{T}]$. In the sequel, we will review strengthened versions of the above calculus, as well as several of their more recent applications. 
2. Holomorphic semigroups. In [dL1], the study of well-bounded operators and $C^{(1)}$ scalar operators is approached via functional calculi for generators of holomorphic semigroups which are uniformly bounded on the right-hand half-plane $\mathbb{C}^{+}$. In [dL2], wellboundedness is analysed for generators $A$ of holomorphic semigroups of growth $O(|z|)$ on $r+\mathbb{C}^{+}, r>0$, using the inverse Laplace transform of $z^{-2} e^{-z A}$. Inspired by [dL1], a fairly general functional calculus is established in [GP], for holomorphic semigroups with polynomial growth in $\mathbb{C}^{+}$. The construction of such a calculus uses fractional derivation:

Let $C_{c}^{\infty}[0, \infty)$ denote the algebra of infinitely differentiable functions on $[0, \infty)$ with compact support. For $f$ in $C_{c}^{\infty}[0, \infty)$ let $W^{-\nu} f$ denote the fractional integral of $f$ of order $\nu>0$,

$$
W^{-\nu} f(t):=\frac{1}{\Gamma(\nu)} \int_{t}^{\infty}(t-s)^{\nu-1} f(s) d s, \quad t>0 .
$$

The Weyl fractional derivative $W^{\nu} f$ of $f$, of order $\nu>0$, is then defined by

$$
W^{\nu} f(t):=(-1)^{n} \frac{d^{n}}{d t^{n}} W^{-(n-\nu)} f(t), \quad t>0,
$$

where $n$ is any natural number greater than $\nu$. We have that $W^{\nu} W^{\mu} f=W^{\nu+\mu} f$ for every $\nu, \mu \in \mathbb{R}$. See $[\mathrm{SKM}]$ for instance.

The following spaces of absolutely continuous functions of higher (fractional) degree are defined in [GP], [GMP], see also [T] for the first one. Take $\nu>0$. By $A C^{(\nu)}$ we denote the Banach space obtained as the completion of $C_{c}^{\infty}[0, \infty)$ in the norm

$$
\|f\|_{(\nu)}:=\frac{1}{\Gamma(\nu)} \int_{0}^{\infty}\left|W^{\nu} f(t)\right| t^{\nu-1} d t .
$$

The space $A C_{\exp }^{(\nu)}$ is defined analogously to $A C^{(\nu)}$ just replacing the weight $t^{\nu-1}$ with the weight $e^{u}$ in the integral. We denote the norm of $A C_{\exp }^{(\nu)}$ by $\|\cdot\|_{(\nu), e}$. Analogously, let $A C_{2,1}^{(\nu)}$ denote the completion of $C_{c}^{\infty}[0, \infty)$ in the norm

$$
\|f\|_{(\nu) ; 2,1}:=\int_{0}^{\infty}\left[\int_{y}^{2 y}\left|W^{\nu} f(x) x^{\nu}\right|^{2} \frac{d x}{x}\right]^{1 / 2} d y .
$$

As a matter of fact, $f^{(\nu)}$, as defined in Section 1, exists for every $f$ in each of the above spaces. Then we put $W^{\nu} f:=(-1)^{[\nu]} f^{(\nu)}$.

Among other properties, the spaces $A C^{(\nu)}$ and $A C_{2,1}^{(\nu)}$ are invariant under the change of variable $t \mapsto t^{\theta}, \theta>0$ [GP, Proposition 3.5 and Proposition 3.9], and $A C_{\exp }^{(\nu+1 / 2)} \subset$ $A C^{(\nu+1 / 2)} \subset A C_{2,1}^{(\mu)} \subset A C^{(\mu)}$ if $\nu>\mu>0$ where the inclusions are continuous [GP, Proposition 3.7]. Moreover, $A C_{\exp }^{(\nu)}, A C^{(\nu)}$, if $\nu \geq 1$, and $A C_{2,1}^{(\nu)}$, if $\nu>1 / 2$, are Banach algebras with respect to pointwise multiplication. This can be proved using a Leibniz type formula, which seems to be interesting in its own right (see also [T] for $A C^{(\nu)}$ ). The formula is

$$
\begin{aligned}
W^{\nu}(f . g)(x)= & W^{\nu} f(x) g(x)+f(x) W^{\nu} g(x) \\
& -\int_{x}^{\infty} \int_{x}^{\infty}\left(\varphi_{t, u}^{\nu-1}\right)^{\prime}(x) W^{\nu} f(t) W^{\nu} g(u) d t d u,
\end{aligned}
$$

for every $\nu>0$ and $f, g$ in the Schwartz class on $[0, \infty)$. Here $\varphi_{t, u}^{\nu}(x)$ is a certain function 
involving the Gaussian hypergeometric function ${ }_{2} F_{1}$. For more details, see [GP, p. 316 and 325], [GMP].

Also, $A C^{(\nu)}$ is a closed subalgebra of the algebra $B V_{\nu}$ formerly considered, for every $\nu \geq 1$, such that $f_{\infty}=0$ for all $f$ in $A C^{(\nu)}$ [GP, Proposition 3.1]. If $1<\mu<\nu$ and $f$ in $B V_{\nu}$ is such that $f_{\infty}=0$ then $W^{\mu} f(t):=\Gamma(\nu-\mu)^{-1} \int_{t}^{\infty}(u-t)^{\nu-\mu-1} d f^{(\nu-1)}(u)$ is already a function. Therefore $f \in \bigcap_{\mu<\nu} A C^{(\mu)}$. Thus we deal with $A C^{(\nu)}$ instead of $B V_{\nu}$, for convenience.

Let $\left(a^{z}\right)_{\Re z>0}$ be an analytic $C_{0}$ semigroup of bounded operators on $X$ with generator $-A$. We will assume additionally that the semigroup, or alternatively the operator $A$, satisfies property $\left(H G_{\alpha}\right)$ for some $\alpha \geq 0$ :

$$
\left\|a^{z}\right\| \leq C_{\alpha}\left(\frac{|z|}{\Re z}\right)^{\alpha}, \quad(\Re z>0),
$$

where $C_{\alpha}$ is some positive constant. (This property has also been considered in relation with integrated semigroups $[\mathrm{ABHN}],[\mathrm{E}]$. It is readily seen that the property implies $\sigma(A) \subset[0, \infty)$.)

Then, if $x \in X, u \in \mathbb{R}$, and $\nu>\alpha$, the inverse Laplace transform of $z^{-(\nu+1)} a^{z} x$,

$$
G^{\nu}(u) x:=\frac{1}{2 \pi i} \int_{\Re z=1} z^{-(\nu+1)} e^{u z} a^{z} x d z,
$$

is well defined in $u \in \mathbb{R}$, with $G^{\nu}(u) \in \mathcal{L}(X), G^{\nu}(u)=0$ if $u \leq 0$, and $\left\|G^{\nu}(u)\right\| \leq C_{\nu} u^{\nu}$ if $u>0$. The kernel $G^{\nu}(u)$ was introduced in [GP], independently of [dL2]. It allows us to establish an explicit functional calculus for $A$ in the following terms, see [GP, Theorem 4.1 and Theorem 6.3].

Theorem 2.1. Let $A$ be as above, satisfying property $\left(H G_{\alpha}\right)$. For every $\nu>\alpha$, the formula

$$
\Phi(f)=\int_{0}^{\infty} W^{\nu+1} f(u) G^{\nu}(u) d u
$$

defines a bounded Banach algebra homomorphism $\Phi: A C^{(\nu+1)} \rightarrow \mathcal{L}(X)$, which is independent of $\nu$, such that $\Phi\left(A C^{(\nu+1)}\right) X$ is dense in $X$ and $\Phi(f) A \subset A \Phi(f)=\Phi(g)$ where $g(u)=u f(u)$, whenever $g, f \in A C^{(\nu+1)}$.

In view of the above result, if $A$ is a closed, densely defined operator on $X$, we say that $A$ has an $A C^{(\nu+1)}$-calculus, where $\nu \geq 0$, if there exists a bounded Banach algebra homomorphism $\Psi: A C^{(\nu+1)} \rightarrow \mathcal{L}(X)$ which satisfies the properties of Theorem 2.2. Clearly, the $A C^{(\nu+1)}$-calculus defined by $\Phi$ looks very much like that given by formula (1.1). Indeed, both calculi are the same: Taking $\mu>\nu>\alpha$, the function $f(u):=\Gamma(\mu+1)^{-1}(\lambda-u)_{+}^{\mu}$, $u>0$, belongs to $A C^{(\nu+1)}$, and it can be seen that $\Phi(f)=G^{\mu}(\lambda)$ [GP, p. 332]. That is, $G^{\nu}(u)=R_{\nu}(u, A)$ for all $\nu>\alpha, u>0$. To complete the picture, note that the existence of an $A C^{(\nu+1)}$-calculus for an operator $A$ entails the existence of a holomorphic $C_{0}$ semigroup $a^{z}$, generated by $-A$, with property $\left(H G_{\nu+1}\right)$ : It is enough to define $a^{z}$ as the image, through such a calculus, of the function $u \mapsto e^{-z u}$, for $z \in \mathbb{C}^{+}$. In short, we have that, for a given closed and densely defined operator $A$ on $X$, the existence of an $A C^{(\nu+1)}$-calculus in $\mathcal{L}(X)$, the existence of a Bochner-Riesz kernel in $\mathcal{L}(X)$ with polynomial growth, and the existence of analytic semigroups in $\mathcal{L}(X)$ with property $\left(H G_{\alpha}\right)$ are 
equivalent. Nevertheless, there is no exact correspondence between the degree of derivation of $A C^{(\nu+1)}$ and the (polynomial) growth in $\left(H G_{\alpha}\right)$, unless the kernel $G^{\nu}(u)$ enjoys some additional properties.

Proposition 2.2 ([GP, Proposition 7.5]). Let $A$ be a closed, densely defined operator on a Banach space. Let $\alpha \geq 1$. Then $A$ has an $A C^{(\nu)}$ calculus for every $\nu>\alpha$ if and only if $-A$ generates a holomorphic $\left(C_{0}\right)$ semigroup $\left(a^{z}\right)_{\Re z>0}$ in $\mathcal{L}(X)$ satisfying property $\left(H G_{\alpha}\right)$ and such that, for any $\nu>\alpha$, the kernel $G^{\nu}(u)$ is continuously differentiable on $(0, \infty)$ with $u^{1-\nu}\left\|\frac{d}{d u} G^{\nu}(u)\right\|$ bounded.

Let us remark that well-boundedness corresponds to the $A C^{(\nu+1)}$-calculus for $\nu=0$. In this sense, the above result is an extension of results in [dL2]. Further, the kernel $G^{\nu}(u)$ can be regarded as a " $\nu$-times integrated decomposition of the identity" [GP], [dL2].

Question. Is it possible to develop a consistent theory of $\nu$-times integrated decompositions of the identity?

The $A C^{(\nu+1)}$-calculus, considered as "well-boundedness of higher degree", and other related concepts are approached in detail in [GMM], for special operators.

REMARK. Assume now that a semigroup $a^{z} \equiv e^{-z A}$ satisfies the following more general condition than $\left(H G_{\alpha}\right)$. This is what we denote by $\left(G_{\alpha}\right)$, where $\alpha \geq 0$ :

$$
\sup _{\Re z \geq 1} \frac{\left\|a^{z}\right\|}{|z|^{\alpha}}<\infty \text {. }
$$

In this case the kernel $G^{\nu}(u)$ is at most exponentially bounded, i.e., $\left\|G^{\nu}(u)\right\| \leq C_{\nu} e^{u}$ for $u>0$, and the formula $\Phi(f)=\int_{0}^{\infty} W^{\nu+1} f(u) G^{\nu}(u) d u$ defines a functional calculus $\Phi: A C_{\exp }^{(\nu+1)} \rightarrow \mathcal{L}(X)$, for $\nu>\alpha$, which enjoys the properties given in Theorem 2.1. Moreover, the equality $G^{\nu}(u)=R_{\nu}(u, A)$ still holds [GP, pp. 327-332].

For a discussion on more variants of properties $\left(G_{\alpha}\right)$ and $\left(H G_{\alpha}\right)$ see [GMP, pp. $\left.390 \mathrm{ff}.\right]$

3. Davies-Helffer-Sjöstrand calculus. In [D1], a functional calculus has been constructed on the basis of the reproducing formula

$$
f(u)=\frac{1}{2 \pi i} \int_{\mathbb{C}} \frac{\partial \tilde{f}}{\partial \bar{z}}(z)(z-u)^{-1} d z d \bar{z}, \quad(u \in \mathbb{R}),
$$

where $f \in C_{c}^{(\infty)}(\mathbb{R})$ and $\tilde{f}$ is an almost-analytic extension to $\mathbb{C}$ of $f$, of (arbitrarily) fixed degree $m \in \mathbb{N}$, such that $\left|\frac{\partial \tilde{f}}{\partial \bar{z}}(z)\right|=O\left(|\Im z|^{m}\right)$ as $|\Im z| \rightarrow 0$. For $n \in \mathbb{N}$, let $\mathcal{A}_{n}$ be the completion of $C_{c}^{(\infty)}(\mathbb{R})$ with respect to the norm

$$
\int_{-\infty}^{\infty}|f(u)|\left(1+u^{2}\right)^{-1 / 2} d u+\int_{-\infty}^{\infty}\left|f^{(n)}(u)\right|\left(1+u^{2}\right)^{(n-1) / 2} d u .
$$

Then $\mathcal{A}_{n}$ is a Banach algebra for pointwise multiplication. Note that $\mathcal{A}_{n}$ resembles the algebra $A C^{(n)}$ of Section 2. Clearly, $\mathcal{A}_{n} \subset A C^{(n)}$. An important difference between them is that $\mathcal{A}_{n}$ is not homogeneous, that is, it is not invariant under dilations. 
Let $A$ now be a closed, densely defined operator on $X$ with $\sigma(A) \subset \mathbb{R}$. Assume that the resolvent function of $A$ satisfies

$$
\left\|(z-A)^{-1}\right\| \leq C \frac{\left(1+|z|^{2}\right)^{\alpha / 2}}{|\Im z|^{\alpha+1}},
$$

for every $z \notin \mathbb{R}$, where $\alpha \geq 0$ is fixed. The Davies calculus is as follows.

Theorem 3.1 ([D1]). Take $n>\alpha$. Then there exists a well defined, bounded Banach algebra homomorphism $\Theta: \mathcal{A}_{n+1} \longrightarrow \mathcal{L}(X)$ given by

$$
\Theta(f)=\frac{1}{2 \pi i} \int_{\mathbb{C}} \frac{\partial \tilde{f}}{\partial \bar{z}}(z)(z-A)^{-1} d z d \bar{z}
$$

for every $f \in C_{c}^{(\infty)}(\mathbb{R})$.

This calculus has received much attention recently. One of its applications is to results about $L^{p}$ spectral independence in a variety of examples (see [D2], [GMP] and references therein).

On the other hand, there is a neat relationship between the calculi $\Theta$ and $\Phi$ of the previous section. Both $\Theta$ and $\Phi$ coincide with the $L^{\infty}$ spectral calculus when $A$ is selfadjoint on a Hilbert space [D1], [GP]. Property $\left(H G_{\alpha}\right)$ implies $\left(R_{\alpha}\right)$ and, reciprocally, if $\sigma(A) \subset[0, \infty)$ then $\left(R_{\alpha}\right)$ implies $\left(G_{n+1}\right)$ for every $\varepsilon+A, \varepsilon>0$, if $n>\alpha$. So we can consider, also in this case, the calculus $\Phi[$ GMP, p. 392]. Then we have the following.

Theorem 3.2 ([GMP, Theorem 2.1]). Let $\left(a^{z}\right)_{\Re z>0}$ be a holomorphic $C_{0}$ semigroup in $\mathcal{L}(X)$ with infinitesimal generator $-A$ such that $\sigma(A) \subset[0, \infty)$. Assume that $A$ satisfies condition $\left(R_{\alpha}\right)$ for some $\alpha \geq 0$. Then $\Theta(f)=\Phi(f)$ for every $f \in C_{c}^{(\infty)}(\mathbb{R})$.

According to the above result, under assumption $\left(H G_{\alpha}\right), \Theta$ and $\Phi$ are different expressions of the same concept. The formulation of the calculus in terms of $\Phi$ admits an improvement of the order of derivation which is very convenient for applications.

4. Automatic extension of the $A C^{(\nu)}$ calculus. Let $\left(a^{z}\right)_{\Re z>0}$ be a holomorphic $C_{0}$ semigroup in $\mathcal{L}(X)$, of generator $-A$, with property $\left(H G_{\alpha}\right), \alpha \geq 0$. For $t \in \mathbb{R}$ and $f$ in $C_{c}^{(\infty)}(\mathbb{R})$ we define $a^{i t} \Phi(f):=\Phi\left(e^{-i t(\cdot)} f\right)$. For $r>0, u \geq 0, \mu=\nu+(1 / 2)$ and $\nu>\alpha$, the operator $a^{i t} G^{\mu}(u):=\frac{1}{2 \pi i} \int_{\Re z=r} z^{-(\mu+1)} a^{z+i t} e^{u z} d z$ does not depend on $r$. Then $a^{i t} \Phi(f)$ can be written as the limit in the operator norm, as $h \rightarrow 0^{+}$, of the operators

$$
\begin{aligned}
a^{i t} \Phi_{h}(f) & :=\frac{1}{h} \int_{0}^{\infty}\left[W^{\mu} f(u)-W^{\mu} f(u+h)\right] a^{i t} G^{\mu}(u) d u \\
& =\frac{1}{2 \pi} \int_{-\infty}^{\infty} \frac{a^{r+i(r s+t)}}{r^{\mu-1}(1+i s)^{\mu}} \int_{0}^{1} e^{-\lambda h r(1+i s)} d \lambda \hat{g}(-r s) d s,
\end{aligned}
$$

where $\widehat{g}$ denotes the Fourier transform of the function $g(u)=W^{\mu} f(u) e^{(r / 2) u} \chi_{[0, \infty)}(u)$ for $u \in \mathbb{R}$. By applying the Cauchy-Schwarz inequality and the Plancherel formula, and taking the infimum we get

$$
\left\|a^{i t} \Phi_{h}(f)\right\| \leq C\left(1+t^{2}\right)^{\frac{\alpha}{2}} \inf _{r>0} \frac{\left(1+r^{2}\right)^{\frac{\alpha}{2}}}{r^{\nu+\alpha}}\left(\int_{0}^{\infty}\left|W^{\nu+\frac{1}{2}} f(u) e^{r u}\right|^{2} d u\right)^{\frac{1}{2}} .
$$


Dividing $(0, \infty)$ into intervals $\left[2^{k}, 2^{k+1}\right)$, the above infimum can be suitably estimated so that, as $h \rightarrow 0^{+}$, we finally obtain

$$
\left\|a^{i t} \Phi(f)\right\| \leq C\left(1+t^{2}\right)^{\frac{\alpha}{2}} \int_{0}^{\infty}\left[\int_{y}^{2 y}\left|W^{\nu+\frac{1}{2}} f(u) u^{\nu}\left(1+u^{2}\right)^{\frac{\alpha}{2}}\right|^{2} d u\right]^{\frac{1}{2}} \frac{d y}{y} .
$$

If $t=0$ in the previous argument, then we get the better estimate

$$
\begin{aligned}
\|\Phi(f)\| & \leq C \inf _{r>0} r^{-\nu}\left(\int_{0}^{\infty}\left|W^{\nu+\frac{1}{2}} f(u) e^{r u}\right|^{2} d u\right)^{\frac{1}{2}} \\
& \leq C \int_{0}^{\infty}\left[\int_{y}^{2 y}\left|W^{\nu+\frac{1}{2}} f(u) u^{\nu}\right|^{2} d u\right]^{\frac{1}{2}} \frac{d y}{y} .
\end{aligned}
$$

(See [GMP, pp. 399, 400], [GP, pp. 330, 341] for the above calculations.) Thus we derive THEOREM 4.1 ([GP, Theorem 6.4]). Assuming that A satisfies the hypothesis $\left(H G_{\alpha}\right)$, the calculus $\Phi$ is in fact a bounded Banach algebra homomorphism $\Phi: A C_{2,1}^{\left(\nu+\frac{1}{2}\right)} \rightarrow \mathcal{L}(X)$, given by

$$
\Phi(f)=\lim _{h \rightarrow 0^{+}} \frac{1}{h} \int_{0}^{\infty} W^{\nu+\frac{1}{2}} f(u) \frac{G^{\nu+\frac{1}{2}}(u-h)-G^{\nu+\frac{1}{2}}(u)}{h} d u,
$$

for every $f \in C_{c}^{(\infty)}(\mathbb{R})$, and provided that $\nu>\alpha$.

(It is possible to prove a similar result to Theorem 4.1 when the operator $A$ is assumed to have property $\left(G_{\alpha}\right)$ [GP, p. 329], [GMP, p. 399].)

From now on we will use also $f(A)$ to refer to the calculus $\Phi$.

5. Fractional powers and some estimates. As said before, the algebra $A C_{2,1}^{\left(\nu+\frac{1}{2}\right)}$ is invariant under the action of the isomorphisms $u \mapsto u^{\theta}, \theta>0$, on $(0, \infty)$ (thus, in the sequel, the symbol $f(A)$ includes general expressions of the form $g\left(A^{\theta}\right)$ ). In particular, the fractional powers $A^{\theta}$ with $\theta>0$ can be defined in a fairly simple way for an operator $A$ with property $\left(H G_{\alpha}\right)$ [GP, pp. 342-344]. We can choose, for instance, to introduce $-A^{\theta}$ as the infinitesimal generator of the holomorphic semigroup $e_{z, \theta}(A)$ where $e_{z, \theta}(u)=$ $\exp \left(-z u^{\theta}\right), u>0, \Re z>0$. Then this semigroup satisfies $\left(H G_{\nu+(1 / 2)}\right)$ for all $\nu>\alpha$. This definition enjoys all good properties that a fractional power is expected to have, and coincides with other standard or usual definitions (under diverse hypotheses). As a remarkable example, we get that $A^{1 / 2}$ equals the square root $\sqrt{A}$ of $A$ when $A$ is the Laplacian operator $\Delta_{n}:=-\frac{\partial^{2}}{\partial x_{1}^{2}}-\frac{\partial^{2}}{\partial x_{2}^{2}}-\cdots-\frac{\partial^{2}}{\partial x_{n}^{2}}$ on $\mathbb{R}^{n}$.

In fact, the example of Laplacian shows that Theorem 4.1 is the best we can obtain concerning the degree of derivation of operating functions: It is well known that $-\Delta_{n}$ generates the Gauss heat semigroup in $L^{1}\left(\mathbb{R}^{n}\right)$ given by convolution with the function $g_{z}(x)=(4 \pi z)^{-n / 2} e^{-\|x\|^{2} /(4 z)},\left(x \in \mathbb{R}^{n} ; z \in \mathbb{C}^{+}\right)$. Further, $\left(g_{z}\right)_{\Re>0}$ is a holomorphic $C_{0}$ semigroup which satisfies $\left(H G_{n / 2}\right)$. Similarly, $-\sqrt{\Delta_{n}}$ generates the Poisson semigroup in $L^{1}\left(\mathbb{R}^{n}\right)$, defined by convolution with the function $p_{z}(x)=c_{n} z\left(z^{2}+\|x\|^{2}\right)^{-(n+1) / 2}$, $\left(x \in \mathbb{R}^{n} ; z \in \mathbb{C}^{+}\right)$, where $c_{n}=\Gamma((n+1) / 2) \pi^{-(n+1) / 2}$. Also, $\left(p_{z}\right)_{\Re z>0}$ is a holomorphic $C_{0}$ semigroup which satisfies $\left(H G_{\alpha_{n}}\right)$ with $\alpha_{n}=(n-1) / 2$, for $n \geq 2$. Suppose, if possible, that in Theorem 4.1 the degree of derivation could be improved up to $\nu+\beta$, 
where $\nu>\alpha$ and $0 \leq \beta<1 / 2$. Then, applying the theorem to $A=\sqrt{\Delta_{n}}$ and the function $e_{z, 2}$, we would obtain that the Gaussian semigroup $e^{-z \Delta_{n}}=e_{z, 2}\left(\sqrt{\Delta_{n}}\right)$ satisfies $\left(H G_{\gamma}\right)$ for all $\gamma=\nu+\beta$ such that $\nu>\alpha_{n}$. In particular we could take $\gamma$ such that $(n-1) / 2+\beta<\gamma<n / 2$. But this is known to be false [ABHN, p. 176].

Besides the above examples, there exist many other heat semigroups in $L^{p}$-spaces, generated by Laplacians or sub-Laplacians on certain Riemann manifolds and Lie groups, satisfying condition $\left(H G_{\alpha}\right)$ [D1], [D2], [D], [P], [T], [GMP]; see also [EK] in connection with integrated cosine functions. (Thus all the results stated in this section, and in sections below, are applicable to these examples.) We have just seen that the $A C_{2,1}^{(\mu)}$ calculus for $\Delta_{n}$ on $\mathbb{R}^{n}$ can be improved in $1 / 2$ order of derivation via the usage of the operator $\sqrt{\Delta_{n}}$ and composition with the mapping $u \mapsto u^{2}$. This is also the case for a suitable sub-Laplacian on the Heisenberg group $\mathbb{H}_{n}$, see [MS], [GMP, p. 409], [GMM]. Since this fact implies the degree of differentiability which is the appropriate one in multiplier theorems, we find the following question interesting.

Question. Let $-A$ be a sub-Laplacian operator on a Lie group $G$ whose generated heat semigroup satisfies condition $\left(H G_{\alpha}\right)$, for some $\alpha>1 / 2$. What kind of assumptions on $A$ and/or $G$ ensure that the semigroup generated by $-\sqrt{A}$ enjoys property $\left(H G_{\alpha-(1 / 2)}\right)$ ?

Pure imaginary powers of type $e^{-i t A}$ are related to ill-posed Cauchy problems [dL3]. The estimate (4.1) gives us immediately the following.

Proposition 5.1 ([GMP, Theorem 3.3]). Suppose that $A$ has property $\left(H G_{\alpha}\right)$, for some $\alpha>0$. Let $t \in \mathbb{R}$. Then $e^{-i t A} f(A) \in \mathcal{L}(X)$ and

$$
\left\|e^{-i t A} f(A)\right\| \leq C\left(1+t^{2}\right)^{\frac{\alpha}{2}} \int_{0}^{\infty}\left[\int_{y}^{2 y}\left|W^{\nu+\frac{1}{2}} f(u) u^{\nu}\left(1+u^{2}\right)^{\frac{\alpha}{2}}\right|^{2} d u\right]^{\frac{1}{2}} \frac{d y}{y}
$$

for every $f \in A C_{2,1}^{\left(\nu+\frac{1}{2}\right)}$, whenever $\nu>\alpha$.

The proposition extends results of [JN], see also [dL3]. More special expressions of the form $e^{-i t A} f(A)$ appear in multiplier theory, or in connection with integrated semigroups, or arise from Cauchy equations.

Corollary 5.2 ([GMP, Corollary 3.5]). If $\Re \nu>\alpha$,

(i) $e^{i t H} H^{-\nu} \varphi(H) \in \mathcal{L}(X)$ and $\left\|e^{i t H} H^{-\nu} \varphi(H)\right\| \leq C_{\nu}\left(1+|t|^{\alpha}\right)$, for each $t \in \mathbb{R}$ and $\varphi$ in $C^{(\infty)}(\mathbb{R})$ such that $\varphi(u)=0$ if $u<\frac{1}{2}$ and $\varphi(u)=1$ if $u>1$.

(ii) $I_{t}^{\nu}(H):=\int_{0}^{t}(t-s)^{\nu-1} e^{i s H} d s \in \mathcal{L}(X)$ and $\left\|I_{t}^{\nu}(H)\right\| \leq C_{\nu} t^{\Re \nu}$, for $t \geq 0$.

(iii) $e^{i t H}(1+H)^{-\nu} \in \mathcal{L}(X)$ and $\left\|e^{i t H}(1+H)^{-\nu}\right\| \leq C_{\nu}\left(1+t^{2}\right)^{\frac{\alpha}{2}}$, for each $t \in \mathbb{R}$.

(iv) $(\sin t H) H^{-1}(1+H)^{-\nu+1} \in \mathcal{L}(X)$ and, for every $t \in \mathbb{R}$ and $0<\varepsilon<\Re \nu$,

$$
\left\|(\sin t H) H^{-1}(1+H)^{-\nu+1}\right\| \leq \begin{cases}C_{\nu}|t|\left(1+|t|^{\Re \nu-1}\right) & \text { if } \Re \nu>1 \text { or } \nu=1, \\ C_{\nu, \varepsilon}|t|^{\Re \nu}\left(1+|t|^{-\varepsilon}\right) & \text { if } 0<\Re \nu \leq 1, \nu \neq 1 .\end{cases}
$$

The proof of the above estimates is not difficult, but requires some subtle calculation, in particular concerning the case (iv) [GMP, p. 402].

Some interesting semigroups satisfy $\left(G_{\alpha}\right)$ but not $\left(H G_{\alpha}\right)[\mathrm{M}]$. In this situation Corollary 5.2 does not work as it stands, but it is still possible to obtain estimates in terms of integrals with respect to polynomial weights [GMP]. 
6. Integral representations of Besov spaces. Assume, once again, that $a^{z}=$ $e^{-z A}$ has property $\left(H G_{\alpha}\right)$. Take $\psi$ in $C_{c}^{(\infty)}(\mathbb{R})$ with support in $\left(2^{-1}, 2\right)$, such that $\sum_{j=-\infty}^{\infty} \psi\left(2^{-j} u\right) \equiv 1$ and then define $\psi_{j}(u)=\psi\left(2^{-j} u\right)$ for $j=1,2, \ldots, \psi_{0}=1-\sum_{j=1}^{\infty} \psi_{j}$. For $j \geq 0$, let $\psi_{j}(A)$ be in $\mathcal{L}(X)$ as defined by the calculus established in Theorem 4.1 (or Theorem 2.1). If $\theta>0$ and $1 \leq q \leq \infty$, let $B^{\theta, q}(X ; A)$ define the Besov space on $X$ associated to $A$ as the subspace of $X$ formed by vectors $x$ such that

$$
\|x\|_{\theta, q}:=\left(\sum_{j=0}^{\infty} 2^{j \theta q}\left\|\psi_{j}(A) x\right\|^{q}\right)^{1 / q}<\infty .
$$

Then $\|x\|_{\theta, q}$ is a norm for which $B^{\theta, q}(X ; A)$ becomes a Banach space (if $q=\infty$ the sum in $j$ must be replaced with $\left.\sup _{j \geq 0} 2^{j \theta}\left\|\psi_{j}(A) x\right\|\right)$. The space $B^{\theta, q}(X ; A)$ does not depend on $\left(\psi_{j}\right)_{j}$, subject to certain conditions. This definition is in $[\mathrm{P}],[\mathrm{Tr}]$ for an operator $A$ with Bochner-Riesz kernels such that $\sup _{u>0} u^{-\alpha}\left\|R_{\alpha}(u, A)\right\|<\infty$. It includes quite a number of interesting examples.

Theorem 6.1 ([G]). If $m>\theta, \nu>\alpha$ and $f \in A C_{2,1}^{(\nu+(1 / 2))} \oplus \mathbb{C}$ is such that $u^{-m} f(u) \in$ $A C_{2,1}^{\left(\nu+\frac{1}{2}\right)}$, then the Besov space $B^{\theta, q}(X ; A)$ coincides with the subspace of $X$ formed by the vectors $x$ such that

$$
\int_{0}^{\infty} t^{-\theta q}\|f(t A) x\|^{q} \frac{d t}{t}<\infty
$$

Moreover, the following norms are all equivalent in $B^{\theta, q}(X ; A)$.

(1) $\left(\sum_{j=0}^{\infty} 2^{j \theta q}\left\|\psi_{j}(A) x\right\|^{q}\right)^{1 / q}$ where $\left(\psi_{j}\right)_{j=0}^{\infty}$ is as above.

(2) $\|x\|+\left(\int_{0}^{\infty} t^{-\theta q}\|f(t A) x\|^{q} \frac{d t}{t}\right)^{\frac{1}{q}}$.

(3) $\|x\|+\left(\int_{0}^{\infty} t^{-\theta q} \sup _{0 \leq s \leq t}\|f(s A) x\|^{q} \frac{d t}{t}\right)^{\frac{1}{q}}$.

REMARKs. (i) The theorem also holds for $q=\infty$. In this case the norms given by $L^{q}$-integrals with respect to the measure $d t / t$ need to be replaced with the sup-norm on $t>0$, as usual. Originally, the theorem is proved in $[\mathrm{G}]$ under the assumption that $A$ is an operator for which the Bochner-Riesz kernels satisfy $\sup _{u>0} u^{-\alpha}\left\|R_{\alpha}(u, A)\right\|<$ $\infty$, but the arguments work exactly equal for semigroups with $\left(H G_{\alpha}\right)$. The proof of the theorem follows standard ideas, and mainly relies on the sub-homogeneity of the functional calculus. As a consequence of the theorem, we have that the norms (1) - (3) are interpolation norms $[\mathrm{BB}]$.

(ii) Through an obvious, suitable choice of functions $f \in A C_{2,1}^{\left(\nu+\frac{1}{2}\right)}$, Theorem 6.1 yields the following equivalent norms in the Besov space (up to addition of $\|x\|$ ):

- $\left(\int_{0}^{\infty} t^{-\theta q}\left\|\left(e^{-t \sqrt{A}}-I\right)^{m} x\right\|^{q} \frac{d t}{t}\right)^{1 / q}$, for $m>\theta$;

- $\left(\int_{0}^{\infty}\left(t^{m-\theta}\left\|(\sqrt{A})^{m} e^{-t \sqrt{A}} x\right\|\right)^{q} \frac{d t}{t}\right)^{1 / q}$, for $m>\theta$; 
- $\left(\int_{0}^{\infty}\left(t^{m-\frac{\theta}{2}}\left\|A^{m} e^{-t A} x\right\|\right)^{q} \frac{d t}{t}\right)^{1 / q}$, for $m>\theta / 2$,

and similar others.

These are norms usually considered in definitions of Besov spaces. The fact that all of them are equivalent is well known for the Laplacian $A=\Delta_{n}$ on $X=L^{p}\left(\mathbb{R}^{n}\right)$, for example, but it is not clear in general. Further, when such an equivalence holds, then it is shown using ad hoc methods [CS], [JN], [S], [G] and references therein. In this way, Theorem 6.1 unifies and extends quite a number of results in the literature.

(iii) With some more work it is possible to give another equivalent norm in compact, integral form:

$$
\left(\int_{0}^{\infty} t^{(m-\theta) q}\left\|(I+A)^{m} e^{-t A} x\right\|^{q} e^{-t} \frac{d t}{t}\right)^{\frac{1}{q}},
$$

where $m>\theta$. This extends analogous results for $\Delta_{n}$ on $\mathbb{R}^{n}[\mathrm{~F}]$, and for sub-Laplacians on stratified groups $[\mathrm{S}]$.

7. Sectorial operators. The calculus $\Phi$ can be approximated by standard holomorphic functional calculus, see [GP, p. 328]. Next, we will see another form of such a relationship.

For $\tau$ such that $0<\tau<\pi$, set $S_{\tau}=\{\lambda \in \mathbb{C} \backslash\{0\}:|\arg (\lambda)|<\tau\}$, where $\arg (\lambda)$ is the argument of $\lambda$ which takes values in $[-\pi, \pi)$. Let $H^{\infty}\left(S_{\tau}\right)$ be the usual Banach algebra of bounded analytic functions on $S_{\tau}$. Let $\mathcal{A}_{b}\left(S_{\tau}\right)$ denote the Banach subalgebra of $H^{\infty}\left(S_{\tau}\right)$ formed by the functions which are also continuous on $\overline{S_{\tau}} \backslash\{0\}$. Set $\psi(\lambda):=\lambda(1+\lambda)^{-2}$, if $\lambda \in S_{\tau}$. For $\delta>0$, we define $\mathcal{A}_{0}^{\delta}\left(S_{\tau}\right)$ as the subalgebra of all functions $f$ of $\mathcal{A}_{b}\left(S_{\tau}\right)$ for which $f(\lambda) \psi^{-\delta}(\lambda) \rightarrow 0$ as $|\lambda| \rightarrow \infty$ or $|\lambda| \rightarrow 0$. Put $\mathcal{D} \mathcal{R}\left(S_{\tau}\right):=\bigcup_{\delta>0} \mathcal{A}_{0}^{\delta}\left(S_{\tau}\right)$, $\mathcal{F}\left(S_{\tau}\right):=\bigcup_{\delta>0} \psi^{-\delta} \cdot \mathcal{A}_{b}\left(S_{\tau}\right)$. Certainly, $\mathcal{D} \mathcal{R}\left(S_{\tau}\right) \subset H^{\infty}\left(S_{\tau}\right) \subset \mathcal{F}\left(S_{\tau}\right)$.

Recall that a closed, one-to-one, operator $A$ with dense domain and dense range in a Banach space $X$ is said to be sectorial of type $\rho$ if $\sigma(A) \in \overline{S_{\rho}}$ and, for every $\tau>\rho$, $\left\|(z-A)^{-1}\right\| \leq C_{\tau}|z|^{-1}$ whenever $z \in \mathbb{C} \backslash S_{\tau}$. Then the Dunford-Riesz integral defines an algebra homomorphism from $\mathcal{D} \mathcal{R}\left(S_{\tau}\right)$ into $\mathcal{L}(X)$, which can be extended from $\mathcal{F}\left(S_{\tau}\right)$ into the linear space $\mathcal{C}(X)$ of closed operators on $X$. The resulting mapping, say $\Upsilon$, becomes a functional calculus in a precise sense [CDMY]. In general the $H^{\infty}$ calculus $\Upsilon$ provides us with operators which are not necessarily bounded. When a sectorial operator $A$ is of type 0 the existence of the (bounded) $H^{\infty}$-calculus $H^{\infty}\left(S_{\tau}\right) \stackrel{\Upsilon}{\rightarrow} \mathcal{L}(X)$ is characterized in terms of Mikhlin-Hörmander multipliers as follows.

For $\alpha>0$, let $\Lambda_{\infty, 1}^{\alpha}\left(\mathbb{R}^{+}\right)$denote the Besov space formed by all bounded continuous functions $F$ on $\mathbb{R}^{+}$such that $\|F\|_{\Lambda_{\infty, 1}^{\alpha}}<\infty$, where

$$
\|F\|_{\Lambda_{\infty, 1}^{\alpha}}=\|F\|_{\infty}+\sum_{k=-\infty}^{\infty} 2^{|k| \alpha}\left\|F_{e} * \check{\phi}_{k}\right\|_{\infty} .
$$

Here, $F_{e}(s):=F\left(e^{s}\right), s \in \mathbb{R}$, and $\left\{\phi_{k}\right\}_{k}$ is a suitable family of functions in $C_{c}(\mathbb{R})$, see [CDMY, p. 73]. The space $\Lambda_{\infty, 1}^{\alpha}\left(\mathbb{R}^{+}\right)$is a Banach algebra for pointwise multiplication.

TheOREM 7.1 ([CDMY, Theorem 4.10]). Let $A$ be a sectorial operator of type 0. Then the following are equivalent. 
(i) A admits a bounded $H^{\infty}$ calculus $\Upsilon$ on $S_{\tau}$ for all $\tau>0$, and there exist $\alpha, C>0$ such that

$$
\|\Upsilon(f)\| \leq C \tau^{-\alpha}\|f\|_{\infty}, \quad \tau>0, f \in H^{\infty}\left(S_{\tau}\right) .
$$

(ii) There exists a bounded algebra homomorphism $\Lambda_{\infty, 1}^{\alpha}\left(\mathbb{R}^{+}\right) \longrightarrow \mathcal{L}(X)$.

This result is used in [D] to establish Mikhlin-Hörmander multiplier theorems in $L^{p}(G)$ with $1<p<\infty$, for sub-Laplacians on homogeneous, nilpotent Lie groups $G$. The proof uses that the semigroup generated by the sub-Laplacian in $L^{1}(G)$ satisfies condition $\left(H G_{\alpha}\right)$ for certain $\alpha>0$. Let us now assume that $-A$ generates a semigroup in $\mathcal{L}(X)$ with $\left(H G_{\alpha}\right)$. Then, for $n>\alpha$ and $z \notin \mathbb{R},\left\|(z-A)^{-1}\right\|=\left\|\Phi\left((z-u)^{-1}\right)\right\| \leq C \|\left((z-u)^{-1} \|_{(n+1)}=\right.$ $\frac{1}{n !} \int_{0}^{\infty} u^{n}|z-u|^{-(n+2)} d u=|z|^{-1}(n !)^{-1} \int_{0}^{\infty} s^{n}\left|s-e^{i \arg (z)}\right|^{-(n+2)} d s \equiv C|z|^{-1}$. Hence, $A$ is sectorial. Note that Theorem 7.1 does not hold in this case, in general (since there is no Mikhlin-Hörmander multiplier results on $L^{1}$ ). Nevertheless, it is possible to establish a result somehow similar to Theorem 7.1, as we will explain later on.

The link between (i) and (ii) in Theorem 7.1 is given by the inclusion $H^{\infty}\left(S_{\tau}\right) \hookrightarrow$ $\Lambda_{\infty, 1}^{\alpha}\left(\mathbb{R}^{+}\right)$, which can be derived from the Paley-Wiener theorem. We now look at this inclusion more closely. Suppose that $h$ is a locally integrable function on $\mathbb{R}^{+}$for which the derivative $h^{(\alpha)}$ (of Introduction) exists. Let $\mathcal{M}_{\infty}^{(\alpha)}$ denote the completion of $C^{(\infty)}\left(\mathbb{R}^{+}\right)$in the norm $\|h\|_{\infty, \alpha}:=\|h\|_{\infty}+\left\|t^{\alpha} h^{(\alpha)}(t)\right\|_{\infty}$, see [CGT] for similar spaces and comments about them. Using the Leibniz rule $(2.1)$ we find that $\mathcal{M}_{\infty}^{(\alpha)}$ is a Banach algebra with respect to pointwise multiplication. The connection of these algebras and those of absolutely continuous functions of Section 2 is given by the fact that $f g \in \mathcal{M}_{2,1}^{(\alpha)}$ for every $f \in \mathcal{M}_{\infty}^{(\alpha)}$ and $g \in \mathcal{M}_{2,1}^{(\alpha)}$ whenever $\alpha>1 / 2$. Here $\mathcal{M}_{2,1}^{(\alpha)}$ is formed by all functions $g$ of $A C_{2,1}^{(\alpha)}$ such that $\int_{0}^{\infty}\left(\int_{t}^{2 t}|f(s)|^{2}(d s / s)\right)^{1 / 2}(d t / t)<\infty$.

Proposition 7.2 ([GM2]). Let $\beta>\alpha>0$ and let $0<\tau<\pi$. Then

$$
\mathcal{A}_{b}\left(S_{\tau}\right) \hookrightarrow \mathcal{M}_{\infty}^{\beta} \hookrightarrow \Lambda_{\infty, 1}^{\alpha}\left(\mathbb{R}^{+}\right) \hookrightarrow \mathcal{M}_{\infty}^{\alpha},
$$

where the inclusions are continuous. In the first one, the inequality $\|h\|_{\infty,(\beta)} \leq C \tau^{-\beta}\|h\|_{\infty}$ holds, for every $h \in \mathcal{A}_{b}\left(S_{\tau}\right)$.

The first inclusion relies on a sort of Cauchy formula for fractional derivatives. The second and third inclusions can be established appealing to the fractional Hadamard operator $\left(u \frac{d}{d u}\right)^{\alpha}$.

It has been pointed out in [GM1] that the, generally unbounded, operators obtained from the $H^{\infty}$ calculus can always be regarded as regular quasimultipliers in the sense defined in [Es]. Going through the main aspects of quasimultiplier theory would take us too far here. So we next give only a partial statement. Details of proofs are rather involved. They will appear in a forthcoming paper.

Let $\Phi_{0}$ denote the restriction mapping of $\Phi$ to $\mathcal{M}_{2,1}^{\left(\nu+\frac{1}{2}\right)}$. Let define $a_{0}^{z}:=\Phi_{0}\left(e_{0}^{z}\right)$, where $e_{0}^{z}(u)=u^{z} e^{-z u}$ for $u \in \mathbb{R}$ and $z \in \mathbb{C}^{+}$. Then $a_{0}^{z}$ is a holomorphic semigroup in $\mathcal{L}(X)$. Put $X_{0}:=\overline{\cup_{\Re z>0} a_{0}^{z} X}$ in $X$. Then we define a Banach space $Y_{0}$ by a certain "extrapolation" method so that $Y_{0}$ contains $X_{0}$ densely. On the other hand, since $\mathcal{M}_{2,1}^{(\alpha)}$ is 
an $\mathcal{M}_{\infty}^{(\alpha)}$-module we can extend the homomorphism $\Phi_{0}$ to the algebra $\mathcal{M}_{\infty}^{(\alpha)}$. Altogether, we get the following.

THEOREM 7.3. Let $-A$ be the generator of a semigroup $\left(a^{z}\right)_{\Re z>0}$ with $\left(H G_{\alpha}\right), \alpha>0$, and let $X_{0}$ be as above. Then there exists a Banach space $Y_{0}$ with $\overline{X_{0}}=Y_{0}$ for which

$$
H^{\infty}\left(S_{\tau}\right) \hookrightarrow M_{\infty}^{\left(\nu+\frac{1}{2}\right)} \stackrel{\Phi_{0}}{\longrightarrow} \mathfrak{L}\left(Y_{0}\right)
$$

is a bounded homomorphism such that

(i) $\left\|\Phi_{0}(h)\right\| \leq C_{\nu, \alpha} \tau^{-\left(\nu+\frac{1}{2}\right)}\|h\|_{\infty}$, for every $h \in H^{\infty}\left(S_{\tau}\right), 0<\tau<\pi$ and $\nu>\alpha$.

(ii) $\Phi_{0}\left((z+\cdot)^{-1}\right)=(z+A)^{-1}$ for every $z \in \mathbb{C} \backslash[0, \infty)$.

We can apply the above result to sub-Laplacians on $X=L^{p}(G)$, where $G$ is a nilpotent group, for $p=1$. If $1<p<\infty$, the theorem given in [D] is better: $Y_{0}$ can be replaced with $X$ itself in the statement (though $\nu+(1 / 2)$ must be changed by $\nu+1$ then). The proof in [D] depends heavily on the structure of $L^{p}$ spaces on $G, 1<p<\infty$. It would be very interesting to understand the mechanism through which $Y_{0}$ can replace $X$ in the above example. More generally,

Question. Under suitable geometric assumptions on $X$, is it possible to derive Theorem 7.3 with $X$ instead of $Y_{0}$ in the statement?

8. Integrated semigroups. Weyl fractional derivation, as defined in Section 2, is well behaved in connection with pointwise multiplication, recall the Leibniz type formula (2.1). There is an analogous formula for the convolution product. This is

$$
\begin{aligned}
\Gamma(\alpha) W^{\alpha}(f * g)(s)= & \int_{0}^{s} W^{\alpha} g(r) \int_{s-r}^{s}(t+r-s)^{\alpha-1} W^{\alpha} f(t) d t d r \\
& -\int_{s}^{\infty} W^{\alpha} g(r) \int_{s}^{\infty}(t+r-s)^{\alpha-1} W^{\alpha} f(t) d t d r,
\end{aligned}
$$

where $\alpha>0, s>0$, and $f, g \in C_{c}^{(\infty)}[0, \infty)$ [M1]. Then the integral $q_{\alpha}(f):=$ $\int_{0}^{\infty}\left|W^{\alpha} f(u)\right| u^{\alpha} d u$ defines a norm such that $q_{\alpha}(f * g) \leq C_{\alpha} q_{\alpha}(f) q_{\alpha}(g)$ for every $f, g \in$ $C_{c}^{(\infty)}[0, \infty)$. Let $\mathcal{T}^{(\alpha)}$ denote the Banach algebra obtained as the completion of $C_{c}^{(\infty)}[0, \infty)$ in this norm.

We define a distribution semigroup of order $\alpha$ and growth $u^{\alpha}$ to be any bounded algebra homomorphism $\Theta: \mathcal{T}^{(\alpha)} \rightarrow \mathcal{L}(X)$ such that $\overline{\Theta\left(\mathcal{T}^{(\alpha)}\right) X}=X$ [M2], [GM1].

A family $\left(T_{\alpha}(t)\right)_{t>0}$ in $\mathcal{L}(X)$ is said to be an $\alpha$-times integrated semigroup if $T_{\alpha}(0)=0$ and

$$
T_{\alpha}(t) T_{\alpha}(s)=\frac{1}{\Gamma(\alpha)}\left(\int_{s}^{t+s}-\int_{0}^{t}\right)(t+s-r)^{\alpha-1} T_{\alpha}(r) d r,
$$

for every $s, t \geq 0$. We say that $T_{\alpha}(t)$ is of growth $t^{\alpha}$ if $\left\|T_{\alpha}(t)\right\| \leq C t^{\alpha}$, for every $t>0$ $[\mathrm{AK}],[\mathrm{H}]$.

Distribution semigroups and integrated semigroups have been introduced to deal with non-bounded operators appearing in ill-posed Cauchy problems. It has been proved that distribution semigroups of integer order $n$ and growth $u^{n}$ and $n$-times integrated semigroups of growth $t^{n}$ coincide [AK], see also [dL3]. This equivalence has been extended to fractional order in [M2]. 
THEOREM 8.1. Distribution semigroups of order $\alpha$ and growth $u^{\alpha}$ are in bijective correspondence with $\alpha$-times integrated semigroups of growth $t^{\alpha}$. Such a bijection is implemented by the formula

$$
\Theta(f) x=\int_{0}^{\infty} W^{\alpha} f(t) T_{\alpha}(t) x d t \quad\left(x \in X ; f \in \mathcal{T}^{(\alpha)}\right),
$$

where $\Theta$ is the distribution semigroup corresponding to the integrated semigroup $T_{\alpha}(t)$ and viceversa.

REMARKs. (1) Theorem 8.1 is given in [M2] in a fairly more complete and general form. In particular the theorem holds for a class of weights quite larger than $u^{\alpha}$, so that it also extends the results of [W] for the exponential case.

(2) It is known that there are also theories about distribution groups and integrated groups. The theorem above also holds in this context [M2], [GM1]. The theory developed in [GM1] enphasizes distribution groups and integrated groups regarded as quasimultipliers.

\section{References}

[AK] W. Arendt and H. Kellerman, Integrated solutions of Volterra integro-differential equations and applications, in: Integrodiff. Eq., Proc. Conf. Trento 1987, G. Da Prato and M. Iannelli (eds.), Pitman Res. Notes Math. 190, Longman, Harlow 1987, 21-51.

[ABHN] W. Arendt, C. J. K. Batty, M. Hieber and Neubrander, Vector-valued Laplace Transforms and Cauchy Problems, Monographs in Math. 96, Birkhäuser, 2001.

[BB] P. L. Butzer and H. Berens, Semi-groups of Operators and Approximation, SpringerVerlag, New York, 1967.

[BNT] P. L. Butzer, R. J. Nessel and W. Trebels, Multipliers with respect to spectral measures in Banach spaces and approximation I, J. Appr. Th. 8 (1973), 335-356.

[CGT1] A. Carbery, G. Gasper and W. Trebels, Radial Fourier multipliers of $L^{p}\left(\mathbb{R}^{2}\right)$, Proc. Nat. Acad. Sci. USA 81 (1984), 3254-3255.

[CGT2] A. Carbery, G. Gasper and W. Trebels, On localized potential spaces, J. Appr. Th. 48 (1986), 251-261.

[CS] T. Coulhon and L. Saloff-Coste, Semi-groupes d'opérateurs et espaces fonctionnels sur les groupes de Lie, J. Appr. Th. 65 (1991), 176-199.

[CDMY] M. Cowling, I. Doust, A. Mcintosh and A. Yagi, Banach operators with a bounded $H^{\infty}$ functional calculus, J. Austral. Math. Soc. (Series A) 60 (1996), 51-89.

[D1] E. B. Davies, The functional calculus, J. London Math. Soc. 52 (1995), 166-176.

[D2] E. B. Davies, $L^{p}$ spectral independence and $L^{1}$ analyticity, J. London Math. Soc. 52 (1995), 177-184.

[dL1] R. deLaubenfels, Functional calculus for generators of uniformly bounded holomorphic semigroups, Semigroup Forum 38 (1989), 91-103.

[dL2] R. deLaubenfels, Unbounded well-bounded operators, strongly continuous semigroups and the Laplace transform, Studia Math. 103 (1992), 143-159.

[dL3] R. deLaubenfels, Existence Families, Functional Calculi and Evolution Equations, Lecture Notes in Math. 1570, Springer, Berlin, 1994. 
[D] X. T. Duong, From the $L^{1}$ norms of the complex heat kernels to a Hörmander multiplier theorem for sub-Laplacians on nilpotent Lie groups, Pacific J. Math. 173 (1996), 413-424.

[E] O. El Mennaoui, Trace des semi-groupes holomorphes singuliers à l'origine et comportement asymptotique, Thèse, Besançon, 1992.

[EK] O. EL Mennaoui and V. Keyantuo, Trace theorems for holomorphic semigroups and the second order Cauchy problem, Proc. Amer. Math. Soc. 124 (1996), 1445-1458.

[Es] J. Esterle, Quasimultipliers, representations of $H^{\infty}$, and the closed ideal problem for commutative Banach algebras, in: Radical Banach Algebras and Automatic Continuity, Proc. Long Beach 1981, Lecture Notes in Math. 975, Springer, Berlin 1983, 66-162.

[F] T. M. Flett, Temperatures, Bessel potentials and Lipschitz spaces, Proc. London Math. 22 (1971), 385-451.

[G] J. E. Galé, Sobre espacios de Besov definidos por medias de Riesz, in: Margarita Mathematica en memoria de José J. Guadalupe (L. Español and J. L. Varona, eds.), Serv. Pub. Univ. de la Rioja, Logroño, 2001, 235-246.

[GM1] J. E. Galé and P. J. Miana, One parameter groups of regular quasimultipliers, J. Funct. Anal. 237 (2006), 1-53.

[GM2] J. E. Galé and P. J. Miana, $H^{\infty}$ calculus and Mikhlin-type multiplier conditions, Canadian J. Math., to appear.

[GP] J. E. Galé and T. Pytlik, Functional calculus for infinitesimal generators of holomorphic semigroups, J. Funct. Anal. 150 (1997), 307-355.

[GMM] J. E. Galé, P. J. Miana and D. Müller, Extensions of well-boundedness and $C^{m}$ scalarity, Int. Eq. Oper. Th., to appear.

[GMP] J. E. Galé, P. J. Miana and T. Pytlik, Spectral properties and norm estimates associated to the $C_{c}^{(k)}$-functional calculus, J. Operator Th. 48 (2002), 385-418.

[GT] G. Gasper and W. Trebels, A characterization of localized Bessel potential spaces and applications to Jacobi and Hankel multipliers, Studia Math. 65 (1979), 243-278.

$[\mathrm{H}] \quad$ M. Hieber, Laplace transforms and $\alpha$-times integrated semigroups, Forum Math. 3 (1991), 595-612.

[HP] E. Hille and R. S. Phillips, Functional Analysis and Semigroups, Amer. Math. Soc. Colloq. 31, Providence, 1957, revised ed., 1974.

[JN] A. Jensen and S. Nakamura, Mapping properties of functions of Schrödinger operators between $L^{p}$ spaces and Besov spaces, in: Spectral and Scattering Theory and Applications, Advanced Studies in Pure Math. 23, 1994, Kinokuniyon Publ., Tokyo, 187-209.

[M1] P. J. Miana, $\alpha$-times integrated semigroups and fractional derivation, Forum Math. 14 (2002), 23-46.

[M2] P. J. Miana, Almost-distributions and integrated families, submitted.

[MS] D. Müller and E. M. Stein, $L^{p}$ estimates for the wave equation on $\mathbb{H}^{n}$, Revista Matemática Iberoamericana 15 (1999), 293-334.

[M] S. Mustapha, Multiplicateurs de Mikhlin pour une classe particulière de groupes nonunimodulaires, Ann. Inst. Fourier Grenoble 48 (1998), 957-966.

[P] J. Peetre, New Thoughts on Besov Spaces, Duke Univ. Math. Series I, Durham, 1976.

[S] K. Saka, Besov spaces and Sobolev spaces on nilpotent Lie groups, Tohoku Math. J. 31 (1979), 383-437.

[SKM] S. G. Samko, A. A. Kilbas and O. I. Marichev, Fractional Integrals and Derivatives. Theory and Applications, Gordon and Breach, New York, 1993. 
[T] W. Trebels, Some Fourier multiplier criteria and spherical Bochner-Riesz kernel, Rev. Roumaine Math. Pures Appl. 20 (1975), 1173-1185.

[Tr] H. Triebel, Fourier Analysis and Function Spaces, Teubner, Leipzig, 1977.

[W] S. W. Wang, Quasi-distribution semigroups and integrated semigroups, J. Funct. Anal. 146 (1997), 352-381. 
